\title{
Effective Gravitational "Constant" in Scalar-(Curvature)Tensor and Scalar-Torsion Gravities
}

\author{
Laur Järv \\ Institute of Physics, University of Tartu, W. Ostwaldi 1, 50411 Tartu, Estonia; laur.jarv@ut.ee \\ Academic Editors: Mariusz P. Dąbrowski, Manuel Krämer and Vincenzo Salzano \\ Received: 1 February 2017; Accepted: 18 April 2017; Published: 24 April 2017
}

\begin{abstract}
In theories where a scalar field couples nonminimally to gravity, the effective gravitational "constant" becomes dependent on the value of the scalar field. This note first gives a brief review on how the cosmological evolution provides a dynamical stabilization for the gravitational "constant" as the system relaxes towards general relativity in matter dominated and potential dominated regimes for scalar-(curvature)tensor and scalar-torsion gravities. Second part summarizes the radius dependence of the gravitational "constant" around a point mass in the parametrized post-Newtonian formalism for scalar-tensor and multiscalar-tensor gravity.
\end{abstract}

Keywords: scalar-tensor gravity; multiscalar-tensor gravity; scalar-torsion gravity; parametrized post-Newtonian formalism; cosmology; effective Newton's constant

\section{Introduction}

General relativity (GR) which ascribes the effects of gravity to the curvature of spacetime has been highly successful in describing a wide range of phenomena. However, the current account of the evolution and structures of the universe entails attributing roughly $25 \%$ of the overall energy density to a host of invisible unknown particles as dark matter, while further $70 \%$ is taken by the cosmological constant which is way too small to receive a natural explanation as a vaccuum energy density of fundamental fields. This puzzle has motivated an intensive research effort in studying various extensions of general relativity that would leave the small scale and weak gravity regimes intact, but suitably modify the large scale physics with some effects permitted also in the strong gravity regime [1-3].

Perhaps one of the simplest extensions of general relativity is to augment the theory by an additional scalar field $\Phi$ nonminimally coupled to curvature $R$. Nonminimal coupling occurs in theories originating from higher dimensions or braneworld setups, it arises due to quantum corrections, and is used in models of inflation (e.g., Higgs inflation), dark energy, or dark matter, and comes about in mathematically equivalent reformulations of $f(R), f(R, \Phi)$ theories [1,2]. Several nonminimally coupled scalar fields can be similarly motivated, while they also appear in mathematically equivalent reformulations of $f\left(R, \square^{i} R, \nabla_{\mu} R \nabla^{\mu} R, \mathcal{G}\right)$, hybrid metric-Palatini $f(R, \mathcal{R})$, nonlocal $f\left(R, \square^{-i} R\right)$, etc., gravities. Nonminimally coupled scalar field modifies GR by dynamically determining the value of the gravitational "constant", i.e., how strongly the matter fields (energy-momentum) affect geometry (the metric field).

Teleparallel equivalent of general relativity (TEGR) assumes Weitzenböck connection instead of Levi-Civita connection and uses torsion instead of curvature to describe gravity [4]. Despite the mathematical and conceptual differences, its predictions are identical to those of general relativity. However, introducing a nonminimal coupling between the torsion $T$ and a scalar field $\Phi$ in the action leads to a theory distinct from a scalar nonminimally coupled to curvature [4]. Nevertheless, the nonminimal scalar still plays the dynamical part of the gravitational "constant", setting the coupling between matter fields (energy-momentum) and geometry (now described by the tetrad field). 
Modified theories can lead to the violation of the equivalence principle and to variable fundamental constants, subject to dedicated experimental investigations (see Refs. [5-9] for reviews and further literature). The theories with a scalar nonminimally coupled to gravity offer a useful testbed here, since as the scalar is universally coupled to all matter fields, the universality of free fall still holds, and the variation is in the gravitational "constant" only.

Starting with a short outline of scalar-curvature, multiscalar-curvature, and scalar-torsion gravities, the present note reviews two topics. First, Section 3 indicates how the cosmological evolution can stabilize the value of the gravitational "constant" in these theories, and gives the approximate behavior in time depending on the model parameters. Then Section 4 summarizes how around a point mass the effective gravitational "constant" can become a function of the distance in scalar-curvature and multiscalar-curvature theories, and how such effect can be mitigated. Finally, Section 5 mentions the latest observational constraints and Section 6 summarizes the conlusions.

\section{Nonminimally Coupled Scalar Field}

\subsection{Scalar-(Curvature)Tensor Gravity}

The most general form of the action for one scalar field $\Phi$ nonminimally coupled to curvature, and without derivative couplings can be written as [10]

$$
S=\frac{1}{2 \kappa^{2}} \int d^{4} x \sqrt{-g}\left\{\mathcal{A}(\Phi) R-\mathcal{B}(\Phi) g^{\mu v} \partial_{\mu} \Phi \partial_{\nu} \Phi-2 \ell^{-2} \mathcal{V}(\Phi)\right\}+S_{\text {matter }}\left[e^{2 \alpha(\Phi)} g_{\mu \nu}, \chi\right]
$$

The two dimensionful constants $\kappa^{2}, \ell$ have been introduced to make $\Phi$ dimensionless, $\chi$ stands for the matter fields. The value of the gravitational "constant", which occurs in the Einstein's equation and determines how the matter energy-momentum tensor is related to Einstein's tensor, is modified by the contribution of the scalar field, $G=\frac{\kappa^{2}}{8 \pi \mathcal{A}(\Phi)}$.

The action (1) is invariant (up to a total divergence term) under a conformal rescaling of the metric (change of frame), $g_{\mu v}=e^{2 \bar{\gamma}(\bar{\Phi})} \bar{g}_{\mu v}$, and redefinition of the scalar field $\Phi=\bar{f}(\bar{\Phi})$. By these transformations two out of the four arbitrary functions $\mathcal{A}(\Phi), \mathcal{B}(\Phi), \mathcal{V}(\Phi), e^{2 \alpha(\Phi)}$ can be fixed at convenience, resulting in different frames and parametrizations of the theory. For instance, in the Jordan frame a parametrization originally used by Brans and Dicke,

$$
S=\frac{1}{2 \kappa^{2}} \int d^{4} x \sqrt{-g}\left\{\Psi R-\frac{\omega(\Psi)}{\Psi} \partial_{\mu} \Psi \partial^{\mu} \Psi-2 \ell^{-2} U(\Psi)\right\}+S_{m}\left[g_{\mu \nu}, \chi\right],
$$

sets out the field value $\Psi$ as the dynamical factor in the gravitational "constant", while the functions $\omega(\Psi)$ and $U(\Psi)$ characterize different theories. Another Jordan frame parametrization

$$
S=\frac{1}{2 \kappa^{2}} \int d^{4} x \sqrt{-g}\left\{(1+f(\varphi)) R-\partial_{\mu} \varphi \partial^{\mu} \varphi-2 \ell^{-2} U(\varphi)\right\}+S_{m}\left[g_{\mu v}, \chi\right]
$$

makes the scalar kinetic term canonical, while $f(\varphi)$ describes deviations from the gravitational "constant" reference value. Despite many different possible parametrizations, all physical observables are in fact independent of the parametrization, and can be expressed in terms of quantities invariant under conformal transformations and scalar field redefinitions [10,11].

\subsection{Multiscalar-(Curvature)Tensor Gravity}

The action (1) can be generalized to include $n$ scalar fields $\Phi=\left\{\Phi^{A}\right\}$ nonminimally coupled to curvature [12,13],

$$
S=\frac{1}{2 \kappa^{2}} \int d^{4} x \sqrt{-g}\left\{\mathcal{A}(\boldsymbol{\Phi}) R-\mathcal{B}_{A B}(\boldsymbol{\Phi}) g^{\mu v} \partial_{\mu} \Phi^{A} \partial_{\nu} \Phi^{B}-2 \ell^{-2} \mathcal{V}(\boldsymbol{\Phi})\right\}+S_{\text {matter }}\left[e^{2 \alpha(\boldsymbol{\Phi})} g_{\mu v}, \chi\right],
$$


while the gravitational "constant" is given by $G=\frac{\kappa^{2}}{8 \pi \mathcal{A}(\boldsymbol{\Phi})}$. An important role in the theory is played by the quantity [12],

$$
\mathcal{F}_{A B} \equiv \frac{2 \mathcal{A} \mathcal{B}_{A B}+3 \frac{\partial \mathcal{A}}{\partial \Phi^{A}} \frac{\partial \mathcal{A}}{\partial \Phi^{B}}}{4 \mathcal{A}^{2}}
$$

which can be interpreted as a generalization of a nonlinear $\sigma$-model metric on the space of fields for the nonminimally coupled case. Negative eigenvalues of this metric indicate that there is a ghost among the scalars, and a zero eigenvalue means one of the scalars is not dynamical and can be integrated out [13].

The action (4) is again invariant (up to a total divergence term) under the conformal rescaling of the metric and redefinitions of the scalar fields. For example, in the case of two fields, it is possible to arrange the fields in the way that one field, $\Psi$, is responsible for the dynamical contribution to the gravitational constant, while the other field $\phi$ is not, so that in the Jordan frame $[13,14]$

$$
S=\frac{1}{2 \kappa^{2}} \int d^{4} x \sqrt{-g}\left(\Psi R-Z(\phi, \Psi) \partial_{\mu} \phi \partial^{\mu} \phi-\frac{\omega(\phi, \Psi)}{\Psi} \partial_{\mu} \Psi \partial^{\mu} \Psi-2 \ell^{-2} U(\phi, \Psi)\right)+S_{m}\left[g_{\mu v}, \chi_{m}\right] .
$$

A particular theory is specified by the functions $Z(\phi, \Psi), \omega(\phi, \Psi), U(\phi, \Psi)$. Since all the couplings between $\Psi$ and $\phi$ cannot be eliminated in general, this action does not reduce to Equation (2), and the second field $\phi$ is still indirectly involved in the nonminimal coupling to curvature. A particularly useful feature of this parametrization is that the field metric (5) is diagonal and the overall number of terms in the equations is lesser. As before, the physical observables are independent of the parametrization [12].

\subsection{Scalar-Torsion Gravity}

Based on the ideas of Einstein (1928) and Møller (1961), the teleparallel equivalent of general relativity (TEGR),

$$
S=\int d^{4} x e\left[\frac{T}{2 \kappa^{2}}\right]+S_{m}\left[e^{a}, \chi\right]
$$

uses Weitzenböck (not Levi-Civita) connection $\stackrel{\mathrm{w}}{\Gamma}_{\nu \mu}^{\lambda} \equiv e_{a}^{\lambda} \partial_{\mu} e_{\nu}^{a}$, which yields zero curvature but nonzero torsion,

$$
\begin{aligned}
& T_{\mu \nu}^{\lambda}=\stackrel{\mathrm{w}}{\Gamma}_{\nu \mu}^{\lambda}-\stackrel{\mathrm{w}}{\Gamma}_{\mu \nu}{ }_{\mu \nu}=e_{a}^{\lambda}\left(\partial_{\mu} e_{v}^{a}-\partial_{\nu} e_{\mu}^{a}\right), \\
& T=\frac{1}{4} T^{\rho \mu \nu} T_{\rho \mu \nu}+\frac{1}{2} T^{\rho \mu \nu} T_{v \mu \rho}-T_{\rho \mu}{ }^{\rho} T_{v}^{v \mu},
\end{aligned}
$$

Here the geometrical variables are the tetrad fields, $\eta_{a b} e^{a}{ }_{\mu} e^{b}{ }_{v}=g_{\mu \nu}, e=\operatorname{det} e^{a}{ }_{\mu}=\sqrt{-g}$ [4]. However, the TEGR equations of motion are equivalent to general relativity, since torsion and curvature scalars differ by a total divergence only, $T=-R-2 \nabla^{\mu} T^{\lambda}{ }_{\mu \lambda}$.

Now if a scalar field is introduced with nonminimal coupling to torsion, i.e., scalar-torsion gravity $[15,16]$,

$$
S=\frac{1}{2 \kappa^{2}} \int d^{4} x e\left(-\mathcal{A}(\Phi) T-\mathcal{B}(\Phi) \partial_{\mu} \Phi \partial^{\mu} \Phi-2 \ell^{-2} \mathcal{V}(\Phi)\right)+S_{m}\left[e^{a}, \chi\right],
$$

the equations of motion are different from the scalar-(curvature)tensor gravity, although the gravitational "constant" will also here depend on the value of the scalar field, $G=\frac{\kappa^{2}}{8 \pi \mathcal{A}(\Phi)}$. The action (10) remains invariant under scalar field redefinitions, but not under conformal transformations. For example, we may redefine the scalar field to give the action a similar form to Equation (3) [16],

$$
S=\frac{1}{2 \kappa^{2}} \int d^{4} x e\left[-(1+f(\varphi)) T-\partial_{\mu} \varphi \partial^{\mu} \varphi-2 \ell^{-2} U(\varphi)\right]+S_{m}\left[e^{a}, \chi\right] .
$$


The equations of motion must be invariant under local Lorentz transformations of the tetrad field, $e^{\prime a}{ }_{\mu}=\Lambda_{b}^{a}(x) e^{b}{ }_{\mu}$, otherwise there will be problems of acausality, etc. [17]. Whereas the TEGR action (7) is locally Lorentz invariant up to a total divergence (boundary) term, the scalar-torsion action is not. A possible way to mend the situation is to include purely inertial spin connection $\omega^{\prime a}{ }_{b \mu}=\Lambda_{c}^{a} \partial_{\mu} \Lambda_{b}^{c}$ as an independent variable (still leading to zero curvature), to delineate the effects of inertia and gravitation [18]. The tetrad corresponding to the flat Friedmann-Lemaitre-Robertson-Walker (FLRW) cosmology is already in a proper form, and the discussion of the next section is not affected by this problem. However, this issue is not so straightforward with the centrally symmetric tetrad [18].

\section{Cosmological Evolution and the Stabilization of the Gravitational "Constant"}

\subsection{Scalar-(Curvature)Tensor Cosmology}

Let us consider flat FLRW cosmology specified by the metric $g_{\mu v}=\operatorname{diag}\left(-1, a(t)^{2}, a(t)^{2}, a(t)^{2}\right)$, and filled with an ideal fluid obeying a barotropic equation of state $\rho=\mathrm{w} p$. For nonminimal scalar-(curvature)tensor gravity in the Jordan frame (2) the cosmlologial evolution is determined by

$$
\begin{aligned}
H^{2} & =\frac{1}{\Psi}\left[\frac{\kappa^{2} \rho}{3}+\frac{\ell^{-2} U(\Psi)}{3}-H \dot{\Psi}+\frac{1}{6} \frac{\dot{\Psi}^{2}}{\Psi} \omega(\Psi)\right], \\
\ddot{\Psi} & =-3 H \dot{\Psi}-\frac{1}{2 \omega(\Psi)+3} \omega^{\prime}(\Psi) \dot{\Psi}^{2}+\frac{\kappa^{2}(1-3 \mathrm{w}) \rho}{2 \omega(\Psi)+3}+\frac{2 \ell^{-2}}{2 \omega(\Psi)+3}\left[2 U(\Psi)-\Psi U^{\prime}(\Psi)\right],
\end{aligned}
$$

where the Hubble parameter $H=\frac{\dot{a}}{a}$ measures the relative expansion of the scale factor $a$, dot signifies derivative with respect to cosmological time, ${ }^{\prime}=\frac{d}{d t}$, and prime indicates derviative with respect to the scalar field, ${ }^{\prime}=\frac{d}{d \Psi}$. We may define the general relativity limit with $\Psi_{\star}$ such that

$$
\dot{\Psi}_{\star}=0, \quad \frac{1}{2 \omega_{\star}+3}=0
$$

and denote $\omega_{\star}=\omega\left(\Psi_{\star}\right), \omega_{\star}^{\prime}=\left.\omega^{\prime}(\Psi)\right|_{\Psi_{\star}}$ etc. In the limit (14) the evolution of the universe converges to look like $\Lambda \mathrm{CDM}$ with the cosmological constant $\Lambda=\ell^{-2} U_{\star}$. If this limit exists and functions as an attractor for the cosmological dynamics, the gravitational "constant" stabilizes to the value $G=\frac{\kappa^{2}}{8 \pi \Psi_{\star}}$ [19]. (Note that it is formally possible to combine the terms in the Friedmann Equation (12) so that it always maintains a fixed gravitational constant, $H^{2}=\frac{\kappa^{2}}{3}\left(\rho+\rho_{D E}\right)$, where all other effects are absorbed into the complicated dynamics of $\rho_{D E}$ [20]. Here we prefer to interpret the $\frac{1}{\Psi}$ factor as a part of $G$, since it accords with the background value of $G_{\text {eff }}$ in the PPN result (31).)

To study the convergence towards GR, let us consider small deviations $x \sim \dot{x} \sim h$ for

$$
\Psi(t)=\Psi_{\star}+x(t), \quad \dot{\Psi}(t)=\dot{x}(t), \quad H(t)=H_{\star}(t)+h(t) .
$$

Expanding the Equations (12) and (13) in series and keeping the leading terms leads to approximate equations which depend on $\omega_{\star}, U_{\star}, \omega_{\star}^{\prime}, U_{\star}^{\prime}$ only [21]. Hence in this analysis different models with different functions $\omega(\Psi), U(\Psi)$ are now reduced to a small set of numbers providing a useful classification.

The equations for $x(t)$ and $h(t)$ are nonlinear and depend implicitly on time, but can still be solved analytically. In the dust matter dominated regime where $\rho \gg U(\Psi), \mathrm{w}=0$, and $H_{\star}(t)=\frac{2}{3\left(t-t_{s}\right)}$ the general solutions are [22]

$$
\Psi(t)=\Psi_{\star} \pm \begin{cases}\frac{1}{t}\left[M_{1} t^{\frac{\sqrt{D_{\star}}}{2}}-M_{2} t^{-\frac{\sqrt{D_{\star}}}{2}}\right]^{2}, & \text { if } \quad D_{\star}>0, \\ \frac{1}{t}\left[M_{1} \ln t-M_{2}\right]^{2}, & \text { if } \quad D_{\star}=0, \\ \frac{1}{t}\left[M_{1} \sin \left(\frac{\sqrt{\left|D_{\star}\right|}}{2} \ln t\right)-M_{2} \cos \left(\frac{\sqrt{\left|D_{\star}\right|}}{2} \ln t\right)\right]^{2}, & \text { if } \quad D_{\star}<0,\end{cases}
$$


while in the potential (cosmological constant) domination regime where $U(\Psi) \gg \rho, H_{\star}=\sqrt{\frac{\ell^{-2} U_{\star}}{3 \Psi_{\star}}}$ the general solutions are [23]

$$
\Psi(t)=\Psi_{\star} \pm \begin{cases}e^{-3 H_{\star} t}\left[M_{1} e^{\frac{1}{2} t \sqrt{C_{\star}}}-M_{2} e^{-\frac{1}{2} t \sqrt{C_{\star}}}\right]^{2}, & \text { if } \quad C_{\star}>0, \\ e^{-3 H_{\star} t}\left[M_{1} t-M_{2}\right]^{2}, & \text { if } \quad C_{\star}=0, \\ e^{-3 H_{\star} t}\left[M_{1} \sin \left(\frac{1}{2} t \sqrt{\left|C_{\star}\right|}\right)-M_{2} \cos \left(\frac{1}{2} t \sqrt{\left|C_{\star}\right|}\right)\right]^{2}, & \text { if } \quad C_{\star}<0 .\end{cases}
$$

Here $M_{1}, M_{2}$ are the constants of integration (to be determined by the initial conditions) while the theory parameters determining the behavior of solutions are

$$
\left.A_{\star} \equiv\left(\frac{1}{2 \omega(\Psi)+3}\right)^{\prime}\right|_{\Psi_{\star}}, \quad D_{\star} \equiv 1+\frac{8}{3} \Psi_{\star} A_{\star}, \quad C_{\star}=\frac{3 \ell^{-2} U_{\star}}{\Psi_{\star}}+4 \ell^{-2} A_{\star}\left(2 U_{\star}-\Psi U_{\star}^{\prime}\right) .
$$

In summary, the solutions dynamically converge towards the GR regime provided the GR limit exists, i.e., the theory allows the value $\Psi_{\star}$ such that $\frac{1}{2 \omega\left(\Psi_{\star}\right)+3}=0$ and

$$
\Psi_{\star} A_{\star}<0, \quad U_{\star}>0, \quad 2 U_{\star}>\Psi_{\star} U_{\star}^{\prime},
$$

in the dust matter dominated era and in the potential dominated era, respectively. The scalar converges to its reference value $\Psi_{\star}$ by either approaching it monotonically or exhibiting damped oscillations. Although the function $\omega$ becomes singular in this limit, analogous behavior of solutions would have also occurred if we had chosen some other frame or parametrization of the scalar field $[10,24]$. Note that the conditions in the dust matter and potential domination eras are unrelated, so the behavior during different eras can be different. In the radiation domination era the damping effects are so strong that the scalar field dynamics freezes without converging to any particular value [19].

Recall that the evolution of the gravitational "constant" follows the inverse of the field $\Psi$. Therefore for any scalar-(curvature)tensor model, it is possible to read out whether GR is an attractor, and if yes, obtain its behavior in cosmological time. Given the observational constraints that the gravitational constant has not changed much over the cosmological timescales $[6,7,25,26]$, only those models can be considered viable, which possess GR as an attractor.

\subsection{Multiscalar-(Curvature)Tensor Cosmology}

The behavior of general multiscalar gravity (4) can be complicated to pursue, but for the two fields case (6) where the kinetic coupling functions depend only one of the fields, e.g., $\omega(\Psi), Z(\Psi)$, it is still possible to obtain some analytic results. The general relativity limit (14) is augmented by $\dot{\phi}_{\star}=0$, $\frac{1}{Z_{\star}}=0$. In the potential dominated case with $H_{\star}=\sqrt{\frac{\ell^{-2} U_{\star}}{3 \Psi_{\star}}}$, the general solution to the approximated equations is [27]

$$
\begin{aligned}
\Psi(t)= & \Psi_{\star}+e^{-3 H_{\star} t}\left[\bar{M}_{1} J_{v}\left(\mu e^{-3 H_{\star} t}\right)-\bar{M}_{2} Y_{v}\left(\mu e^{-3 H_{\star} t}\right)\right]^{2}, \\
\phi(t)= & \phi_{\star}-\frac{\bar{M}_{3}}{6 H_{\star}} e^{-6 H_{\star} t}\left\{\left[\bar{M}_{1} J_{v}\left(\mu e^{-3 H_{\star} t}\right)+\bar{M}_{2} Y_{v}\left(\mu e^{-3 H_{\star} t}\right)\right]^{2}-\right. \\
& {\left[\bar{M}_{1} J_{v+1}\left(\mu e^{-3 H_{\star} t}\right)-\bar{M}_{2} Y_{v+1}\left(\mu e^{-3 H_{\star} t}\right)\right]\left[\bar{M}_{1} J_{v-1}\left(\mu e^{-3 H_{\star} t}\right)-\right.} \\
& \left.\left.\bar{M}_{2} Y_{v-1}\left(\mu e^{-3 H_{\star} t}\right)\right]\right\}+\bar{M}_{4} .
\end{aligned}
$$


Here $J$ and $Y$ are the Bessel functions of the first and second kind, with order and argument given by

$$
v=-\frac{\sqrt{C_{\star}}}{6 H_{\star}}, \quad \mu=\frac{1}{6 H_{\star}} \sqrt{-\frac{\Psi_{\star} A_{\star} Z_{\star}^{\prime} \bar{M}_{3}^{2}}{2 Z_{\star}^{2}}},
$$

$\bar{M}_{1}, \ldots, \bar{M}_{4}$ are the integration constants, while the quantities $A_{\star}, C_{\star}$ were defined in Equation (18). In the limit $t \rightarrow \infty$ the solutions (20) converge to the solutions (17) of the single field case, hence the conditions for a theory to dynamically relax to general relativity, the evolution of the gravitational "constant", and the classification of models are the same. The second scalar field $\phi$ freezes in (22) even faster then $\Psi$.

\subsection{Scalar-Torsion Cosmology}

In scalar-torsion gravity (10) the flat FLRW geometry is specified by the tetrad $e_{\mu}^{a}=\operatorname{diag}(1, a(t), a(t), a(t))$. For a barotropic fluid the ensuing equations of motion are

$$
\begin{aligned}
H^{2} & =\frac{1}{1+f(\varphi)}\left[\frac{\kappa^{2} \rho}{3}+\frac{\ell^{-2} U(\varphi)}{3}+\frac{\dot{\varphi}^{2}}{6}\right], \\
\ddot{\varphi} & =-3 H \dot{\varphi}-3 H^{2} f^{\prime}(\varphi)-\ell^{-2} U^{\prime}(\varphi) .
\end{aligned}
$$

Apart from a different parametrization used for convenience, the equations above are indeed qualitatively different from the equations of scalar-(curvature)tensor cosmology. For example in Equation (24) the scalar field is not sourced by the trace of matter energy momentum, in contrast to Equation (13).

It is still possible to define the general relativity limit

$$
\dot{\phi}_{\star}=0, \quad f^{\prime}\left(\varphi_{\star}\right)=f_{\star}^{\prime}=0, \quad U^{\prime}\left(\varphi_{\star}\right)=U_{\star}^{\prime}=0,
$$

whereby the universe conforms with the $\Lambda$ CDM evolution with the cosmological constant $\Lambda=\ell^{-2} U_{\star}$. The gravitational constant converges then to $G=\frac{\kappa^{2}}{1+f_{\star}}$. Now the interesting question is, can the scalar-torsion cosmology also have GR as an attractor?

We can carry out a similar investigation to the previous case, expand the equations around the GR limit

$$
\varphi(t)=\varphi_{\star}+x(t), \quad \dot{\varphi}(t)=\dot{x}(t), \quad H(t)=H_{\star}(t)+h(t) .
$$

and solve them analytically [16]. If the dust matter dominates, $\rho \gg U$ (and $\left.U_{\star}^{\prime \prime}>0\right), H_{\star}=\frac{2}{3\left(t-t_{s}\right)}$, the solutions are

$$
\varphi(t)=\varphi_{\star}+t^{-\frac{1}{2}}\left(M_{1} J_{v_{\star}}\left(\sqrt{U_{\star}^{\prime \prime}} t\right)+M_{2} Y_{v_{\star}}\left(\sqrt{U_{\star}^{\prime \prime}} t\right)\right) .
$$

If the potential dominates, $U \gg \rho$ while $H_{\star}=\sqrt{\frac{\ell^{-2} U_{\star}}{3\left(1+f_{\star}\right)}}$, then

$$
\varphi(t)=\varphi_{\star}+e^{-\frac{3 H_{\star} t}{2}}\left(M_{1} e^{\frac{3 H_{\star} v_{\star} t}{2}}+M_{2} e^{-\frac{3 H_{\star} v_{\star} t}{2}}\right) .
$$

Here $J$ and $Y u$ are the Bessel functions of the first and second kind, while $M_{1}, M_{2}$ are the integration constants. The quantities

$$
v_{\star}=\sqrt{\frac{1}{4}-\frac{4 f_{\star}^{\prime \prime}}{3}}, \quad v_{\star}=\sqrt{1-\frac{4 f_{\star}^{\prime \prime}}{3}-\frac{4}{3} \frac{\left(1+f_{\star}\right) U_{\star}^{\prime \prime}}{U_{\star}}} .
$$

determine whether the solutions converge or diverge from the GR limit. Again, the convergence to the value $\varphi_{\star}$ is either monotonic or oscillating, depending on the model parameters. In the scalar-torsion 
cosmology the attractor mechanism works and the approximation solutions can be found also in the radiation domination era [16].

Similarly to the scalar-(curvature)tensor case, the behavior of the gravitational "constant" in cosmological time can be read off from the solutions above. So, although scalar-torsion gravity is rather different from scalar-(curvature)tensor gravity, the mechanism to stabilize the gravitational "constant" via cosmological background evolution is also possible here [16].

\section{Effective Gravitational “Constant” around a Point Mass}

The parametrized post-Newtonian (PPN) formalism considers weak quasi-static gravitational field, sourced by a perfect fluid, and compares all quantities in orders of magnitude relative to the matter velocity $v^{i}=\frac{u^{i}}{u^{0}}$ which is assumed to be small. This setup allows versatile comparisons between observations and different gravitational theories in terms of a set of PPN parameters. At the lowest order one determines the effective gravitational "constant", for a point source at the origin (with no pressure or internal energy) given by

$$
g_{00}=-1+2 G_{\text {eff }} \frac{M}{r} .
$$

By construction the background is assumed to be asymptotically Minkowski, which entails $\mathcal{V}_{0}=\mathcal{V}\left(\Phi_{0}\right)=0, \mathcal{V}_{0}^{\prime}=\left.\frac{\partial \mathcal{V}}{\partial \Phi}\right|_{\Phi_{0}}=0$. The subscript zero indicates that the scalar field is taken at its spatially asymptotic value, to be determined by the cosmological evolution. (The cosmological constant or $\mathcal{V}_{0}$ is assumed to be too small to affect local physics.)

\subsection{Scalar-(Curvature)Tensor Gravity}

In the parametrization used in the action (2), the effective gravitational "constant" is $[28,29]$

$$
G_{\text {eff }}=\frac{\kappa^{2}}{8 \pi \Psi_{0}}\left(1+\frac{e^{-m_{\Psi} r}}{2 \omega_{0}+3}\right)
$$

It depends on the distance $r$ from the source, if the scalar field is endowed with nonzero mass,

$$
m_{\Psi} \equiv \frac{1}{\ell} \sqrt{\frac{2 \Psi_{0} U_{0}^{\prime \prime}}{2 \omega_{0}+3}} .
$$

In essence we get here a Yukawa type correction to the Newtonian gravitational potenital. A way to evade contradictions with the observations would be to assume that either $\omega_{0}$ is very large (which is supported by the cosmological attractor mechanism discussed in the previous section), or the mass of the scalar field is very large. (Analogous reasoning applies to the PPN parameters $\gamma$ and $\beta$ as well [29], and can be also rephrased in other frames as parametrizations [10].)

The results above assume that the cosmological attractor mechanism has already stabilized the scalar field, and its background variation in time is negligible for the local observations. However, one may reconsider such assumption [30]. A more sophisticated calculation would then take the cosmological solutions of the previous section as the background for PPN analysis.

\subsection{Multiscalar-(Curvature)Tensor Gravity}

The PPN computation can be also done in theories with multiple nonminimally coupled fields [13,31], although the results are a bit harder to present in a succinct manner. For the two fields case (6), the effective gravitational "constant" reads [13]

$$
G_{\text {eff }}=\frac{\kappa^{2}}{8 \pi \Psi_{0}}\left(1+\frac{\cos ^{2} \vartheta_{+} e^{-m_{+} r}+\cos ^{2} \vartheta_{-} e^{-m_{-} r}}{2 \omega_{0}+3}\right)
$$


Now the radial dependence is mediated by the mass eigenvalues

$$
m_{ \pm}^{2}=\frac{\ell^{-2}}{2 Z_{0}\left(2 \omega_{0}+3\right)}\left(\left.\left(2 \omega_{0}+3\right) \frac{\partial^{2} \mathcal{U}}{\partial \phi^{2}}\right|_{0}+\left.2 \Psi_{0} Z_{0} \frac{\partial^{2} \mathcal{U}}{\partial \Psi^{2}}\right|_{0} \pm B\right)
$$

while the mass eigenvectors are orthogonal to each other with

$$
\cos ^{2} \vartheta_{+}=\frac{1}{2}\left(1+\frac{A}{B}\right), \quad \cos ^{2} \vartheta_{-}=\frac{1}{2}\left(1-\frac{A}{B}\right), \quad \cos ^{2} \vartheta_{+}+\cos ^{2} \vartheta_{-}=1,
$$

where

$$
B=\sqrt{A^{2}+8\left(2 \omega_{0}+3\right) Z_{0} \Psi_{0} \ell^{-2}\left(\left.\frac{\partial^{2} \mathcal{U}}{\partial \phi \partial \Psi}\right|_{0}\right)^{2}}, \quad A=\left.2 \Psi_{0} Z_{0} \ell^{-2} \frac{\partial^{2} \mathcal{U}}{\partial \Psi^{2}}\right|_{0}-\left.\left(2 \omega_{0}+3\right) \ell^{-2} \frac{\partial^{2} \mathcal{U}}{\partial \phi^{2}}\right|_{0} .
$$

The picture is the following. In the two-dimensional space of the fields we may speak of an interaction basis given by $\Psi$ and $\phi$, while $\Psi$ marks a special direction that corresponds to the direct nonminimal coupling to curvature. Another basis can be introduced, given by the eigenvectors of the "mass matrix". For fields that are not of a ghost nature (i.e., the field metrix (5) is positive definite), these eigenvectors are orthogonal to each other. In the formulas above the quantity $\vartheta$ measures the angle between the respective mass eigenvector and the $\Psi$ direction. So, the physical effect does not simply depend on the mass eigenvalues, but is weighted by the angle $\vartheta$. A light mass eigenmode will not have a significant effect if it is almost orthogonal to the $\Psi$ direction. Hence coupling a massless scalar to a massive scalar can greatly reduce the bounds on the parameter $\omega_{0}$. (Note that in the limit where there are no interactions between the scalars, or more precisely $\left.\frac{\partial^{2} \mathcal{U}}{\partial \phi}\right|_{0}=0$, one of the masses $m_{ \pm}$(34) coincides with $m_{\Psi}(32)$, and the biscalar $G_{\text {eff }}$ (33) reduces to the single field case (31).)

\subsection{Scalar-Torsion Gravity}

In contrast to scalar-(curvature)tensor gravities the PPN computation tells that the effective gravitational constant is identically constant in scalar-torsion gravity [32,33]. It is possible to attribute this result to the fact that in scalar-torsion gravity the scalar field is not sourced by matter. However, one must be careful with picking a proper form of the centrally symmetric tetrad [18].

\section{Observational Constraints}

Constraints on the temporal and spatial variation of the gravitational constant can be obtained from a range of phenomena, including Solar System physics, binary pulsars, and cosmology [5-8]. The cosmic microwave background together with the baryon acoustic oscillations restricts the change of the effective gravitational constant between the recombination and the present epochs to be quite small, $\frac{G_{\text {rec }}-G_{\text {now }}}{G_{\text {now }}}<1.9 \times 10^{-3}(95.45 \%$ C.L. $)[25,26]$, while the current Solar System constraints on the time variantion of $G$ are also very strict $[34,35]$. Similarly, the motions of objects in the Solar [36-39] and extra-Solar [40] systems as well as of stars around the massive body in the galactic center [41] are consistent with no significant distance variation of the gravitational constant or a Yukawa type correction. In principle these observations can be employed to constrain the parameters of the models with nonminimally coupled scalar fields (as we did using the PPN parameters [13,29]), however that task remains beyond the scope of the present note.

\section{Conclusions}

In this paper, we considered the extensions of general relativity and teleparallel equivalent of general relativity by nonminimally coupled scalars to curvature or torsion, respectively. The gravitational "constant" in such theories becomes dependent on the scalar field, and thus a dynamical quantity with possible variations in space and time. Contemporary observations in cosmology and astrophysics put rather stringent constraints on such variations. The purpose of 
this short note was to describe the mechanisms whereby a nonminimally coupled scalar can still behave akin to general relativity and conform with the observations.

First, we reviewed how both such classes of theories are endowed with an attractor mechanism, whereby cosmological evolution stabilizes the scalar field, and thus also the gravitational constant. A useful classification of all the models is given by the approximate analytic solutions in cosmological time. The second topic was to indicate some salient features of the effective gravitational "constant" around a point mass source within the parametrized post-Newtonian framework. For scalar-(curvature)tensor and multiscalar-(curvature)tensor gravity, the gravitational "constant" becomes dependent on the distance from the source, mediated by the mass eigenvalues and orientation of the mass eigenvectors in the space of scalar fields. In the scalar-torsion case the gravitational constant is identically constant. As there are a number of gravity theories which are equivalent to some versions of nonminimally coupled scalars, these results can have a wider applicability.

Acknowledgments: The author is very grateful to his collaborators Manuel Hohmann, Piret Kuusk, Erik Randla, Margus Saal, Ott Vilson as well as Alexey Toporensky, with whom most of the work reviewed here was done. This work was supported by the Estonian Ministry of Education and Research via the institutional research funding IUT02-27, and by the European Regional Development Fund through the Center of Excellence "Dark side of the Universe" (TK133).

Conflicts of Interest: The author declares no conflict of interest.

\section{References}

1. Faraoni, V.; Capozziello, S. Beyond Einstein Gravity: A Survey of Gravitational Theories for Cosmology and Astrophysics; Springer: Dordrecht, The Netherlands, 2011; Volume 170.

2. Clifton, T.; Ferreira, P.G.; Padilla, A.; Skordis, C. Modified Gravity and Cosmology. Phys. Rep. 2012, 513, 1-189.

3. Berti, E.; Barausse, E.; Cardoso, V.; Gualtieri, L.; Pani, P.; Sperhake, U.; Stein, L.C.; Wex, N.; Yagi, K.; Baker, T.; et al. Testing General Relativity with Present and Future Astrophysical Observations. Class. Quantum Gravity 2015, 32, 243001.

4. Cai, Y.F.; Capozziello, S.; De Laurentis, M.; Saridakis, E.N. $f(T)$ teleparallel gravity and cosmology. Rep. Prog. Phys. 2016, 79, 106901.

5. Adelberger, E.G.; Gundlach, J.H.; Heckel, B.R.; Hoedl, S.; Schlamminger, S. Torsion balance experiments: A low-energy frontier of particle physics. Prog. Part. Nucl. Phys. 2009, 62, 102-134.

6. Uzan, J.P. Varying Constants, Gravitation and Cosmology. Living Rev. Relativ. 2011, 14, 2.

7. Chiba, T. The Constancy of the Constants of Nature: Updates. Prog. Theor. Phys. 2011, 126, 993-1019.

8. Will, C.M. The Confrontation between General Relativity and Experiment. Living Rev. Relativ. 2014, 17, 4.

9. Burrage, C.; Sakstein, J. A Compendium of Chameleon Constraints. J. Cosmol. Astropart. Phys. 2016, $2016,045$.

10. Järv, L.; Kuusk, P.; Saal, M.; Vilson, O. Invariant quantities in the scalar-tensor theories of gravitation. Phys. Rev. D 2015, 91, 024041.

11. Järv, L.; Kannike, K.; Marzola, L.; Racioppi, A.; Raidal, M.; Rünkla, M.; Saal, M.; Veermäe, H. A frame independent classification of single field inflationary models. Phys. Rev. Lett. 2017, 118, 151302.

12. Kuusk, P.; Järv, L.; Vilson, O. Invariant quantities in the multiscalar-tensor theories of gravitation. Int. J. Mod. Phys. A 2016, 31, 1641003.

13. Hohmann, M.; Järv, L.; Kuusk, P.; Randla, E.; Vilson, O. Post-Newtonian parameter $\gamma$ for multiscalar-tensor gravity with a general potential. Phys. Rev. D 2016, 94, 124015.

14. Kuusk, P.; Järv, L.; Randla, E. Scalar-Tensor and Multiscalar-Tensor Gravity and Cosmological Models. In Algebra, Geometry and Mathematical Physics Springer Proceedings in Mathematics E Statistics; Springer: Berlin/Heidelberg, Germany, 2014; Volume 85, pp. 661-672.

15. Geng, C.Q.; Lee, C.C.; Saridakis, E.N.; Wu, Y.P. “Teleparallel” dark energy. Phys. Lett. B 2011, 704, $384-387$.

16. Järv, L.; Toporensky, A. General relativity as an attractor for scalar-torsion cosmology. Phys. Rev. D 2016, 93, 024051.

17. Li, B.; Sotiriou, T.P.; Barrow, J.D. $f(T)$ gravity and local Lorentz invariance. Phys. Rev. D 2011, 83, 064035. 
18. Krššák, M.; Saridakis, E.N. The covariant formulation of $f(T)$ gravity. Class. Quantum Gravity 2016, 33, 115009.

19. Damour, T.; Nordtvedt, K. General relativity as a cosmological attractor of tensor scalar theories. Phys. Rev. Lett. 1993, 70, 2217-2219.

20. Torres, D.F. Quintessence, superquintessence and observable quantities in Brans-Dicke and nonminimally coupled theories. Phys. Rev. D 2002, 66, 043522.

21. Järv, L.; Kuusk, P.; Saal, M. Potential dominated scalar-tensor cosmologies in the general relativity limit: Phase space view. Phys. Rev. D 2010, 81, 104007.

22. Järv, L.; Kuusk, P.; Saal, M. Scalar-tensor cosmologies with dust matter in the general relativity limit. Phys. Rev. D 2012, 85, 064013.

23. Järv, L.; Kuusk, P.; Saal, M. Scalar-tensor cosmologies with a potential in the general relativity limit: Time evolution. Phys. Lett. B 2010, 694, 1-5.

24. Järv, L.; Kuusk, P.; Saal, M.; Vilson, O. Transformation properties and general relativity regime in scalar-tensor theories. Class. Quantum Gravity 2015, 32, 235013.

25. Ooba, J.; Ichiki, K.; Chiba, T.; Sugiyama, N. Planck constraints on scalar-tensor cosmology and the variation of the gravitational constant. Phys. Rev. D 2016, 93, 122002.

26. Ooba, J.; Ichiki, K.; Chiba, T.; Sugiyama, N. Cosmological constraints on scalar-tensor gravity and the variation of the gravitational constant. arXiv 2017, arXiv:1702.00742.

27. Järv, L.; Kuusk, P.; Randla, E. Biscalar-tensor cosmology in the general relativity limit. Unpublished work.

28. Perivolaropoulos, L. PPN Parameter gamma and Solar System Constraints of Massive Brans-Dicke Theories. Phys. Rev. D 2010, 81, 047501.

29. Hohmann, M.; Järv, L.; Kuusk, P.; Randla, E. Post-Newtonian parameters $\gamma$ and $\beta$ of scalar-tensor gravity with a general potential. Phys. Rev. D 2013, 88, 084054.

30. Arnoulx de Pirey Saint Alby, T.; Yunes, N. Cosmological Evolution and Solar System Consistency of Massive Scalar-Tensor Gravity. arXiv 2017, arXiv:1703.06341 .

31. Randla, E. PPN parameters for multiscalar-tensor gravity without a potential. J. Phys. Conf. Ser. 2014, 532, 012024.

32. Li, J.T.; Wu, Y.P.; Geng, C.Q. Parametrized post-Newtonian limit of the teleparallel dark energy model. Phys. Rev. D 2014, 89, 044040.

33. Chen, Z.C.; Wu, Y.; Wei, H. Post-Newtonian Approximation of Teleparallel Gravity Coupled with a Scalar Field. Nucl. Phys. B 2015, 894, 422-438.

34. Williams, J.G.; Turyshev, S.G.; Boggs, D.H. Progress in lunar laser ranging tests of relativistic gravity. Phys. Rev. Lett. 2004, 93, 261101.

35. Konopliv, A.S.; Asmar, S.W.; Folkner, W.M.; Karatekin, Ö.; Nunes, D.C.; Smrekar, S.E.; Yoder, C.F.; Zuber, M.T. Mars high resolution gravity fields from MRO, Mars seasonal gravity, and other dynamical parameters. Icarus 2011, 211, 401-428.

36. Talmadge, C.; Berthias, J.P.; Hellings, R.W.; Standish, E.M. Model Independent Constraints on Possible Modifications of Newtonian Gravity. Phys. Rev. Lett. 1988, 61, 1159-1162.

37. Sereno, M.; Jetzer, P. Dark matter versus modifications of the gravitational inverse-square law. Results from planetary motion in the solar system. Mon. Not. R. Astron. Soc. 2006, 371, 626-632.

38. Wallin, J.F.; Dixon, D.S.; Page, G.L. Testing Gravity in the Outer Solar System: Results from Trans-Neptunian Objects. Astrophys. J. 2007, 666, 1296-1302.

39. Haranas, I.; Kotsireas, I.; Gómez, G.; Fullana, M.J.; Gkigkitzis, I. Yukawa effects on the mean motion of an orbiting body. Astrophys. Space Sci. 2016, 361, 365.

40. Iorio, L. A model-independent test of the spatial variations of the Newtonian gravitational constant in some extrasolar planetary systems. Mon. Not. R. Astron. Soc. 2007, 376, 1727-1730.

41. Borka, D.; Jovanović, P.; Jovanović, V.B.; Zakharov, A.F. Constraining the range of Yukawa gravity interaction from S2 star orbits. J. Cosmol. Astropart. Phys. 2013, 2013, 050.

(C) 2017 by the author. Licensee MDPI, Basel, Switzerland. This article is an open access article distributed under the terms and conditions of the Creative Commons Attribution (CC BY) license (http:/ / creativecommons.org/licenses/by/4.0/). 